\title{
Coherent quantum dynamics of excitons in monolayer transition metal dichalcogenides
}

\author{
Galan Moody ${ }^{\mathrm{a}, \uparrow}$, Kai Hao ${ }^{\mathrm{a}}$, Chandriker Kavir Dass ${ }^{\mathrm{a}}$, Akshay Singh ${ }^{\mathrm{a}}$, Lixiang Xu ${ }^{\mathrm{a}}$, Kha Tran ${ }^{\mathrm{a}}$, \\ Chang-Hsiao Chen ${ }^{\mathrm{b}}$, Ming-Yang Li ${ }^{\mathrm{c}}$, Lain-Jong Li ${ }^{\mathrm{c}}$, Genevieve Clark ${ }^{\mathrm{d}}$, Gunnar Berghäuser ${ }^{\mathrm{e}}$, \\ Ermin Malic ${ }^{\mathrm{f}}$, Andreas Knorr ${ }^{\mathrm{e}}$, Xiaodong $\mathrm{Xu}^{\mathrm{d}, \mathrm{g}}$, and Xiaoqin $\mathrm{Li}^{\mathrm{a}}$ \\ ${ }^{a}$ Department of Physics, University of Texas at Austin, Austin, Texas 78712, USA \\ ${ }^{\mathrm{b}}$ Department of Automatic Control Engineering, Feng Chia University, Taichung 40724, \\ Taiwan \\ ${ }^{c}$ Physical Science and Engineering Division, King Abdullah University of Science \& \\ Technology (KAUST), Thuwal 23955, Saudi Arabia \\ ${ }^{\mathrm{d}}$ Department of Physics, University of Washington, Seattle, Washington 98195, USA \\ 'Institut f. Theoretische Physik, Nichtlineare Optik und Quantenelektronik, Technische \\ Universitat Berlin, 10623 Berlin, Germany \\ ${ }^{\mathrm{f}}$ Department of Applied Physics, Chalmers University of Technology, Gothenburg, Sweden \\ 'Department of Materials Science and Engineering, University of Washington, Seattle, \\ Washington 98195, USA
}

\begin{abstract}
Transition metal dichalcogenides (TMDs) have garnered considerable interest in recent years owing to their layer thickness-dependent optoelectronic properties. In monolayer TMDs, the large carrier effective masses, strong quantum confinement, and reduced dielectric screening lead to pronounced exciton resonances with remarkably large binding energies and coupled spin and valley degrees of freedom (valley excitons). Coherent control of valley excitons for atomically thin optoelectronics and valleytronics requires understanding and quantifying sources of exciton decoherence. In this work, we reveal how exciton-exciton and exciton-phonon scattering influence the coherent quantum dynamics of valley excitons in monolayer TMDs, specifically tungsten diselenide $\left(\mathrm{WSe}_{2}\right)$, using two-dimensional coherent spectroscopy. Excitation-density and temperature dependent measurements of the homogeneous linewidth (inversely proportional to the optical coherence time) reveal that exciton-exciton and exciton-phonon interactions are significantly stronger compared to quasi-2D quantum wells and 3D bulk materials. The residual homogeneous linewidth extrapolated to zero excitation density and temperature is $\sim 1.6$ $\mathrm{meV}$ (equivalent to a coherence time of $0.4 \mathrm{ps}$ ), which is limited only by the population recombination lifetime in this sample.
\end{abstract}

Keywords: Transition Metal Dichalcogenides, Multi-Dimensional Coherent Spectroscopy, Exciton, Homogeneous Linewidth

\section{INTRODUCTION}

Two-dimensional materials such as graphene and transition metal dichalcogenides (TMDs) have garnered considerable interest in recent years owing to their unique opto-electronic properties and reduced dimensionality. ${ }^{1}$ In the bulk form, group-VIB TMDs $\left(M X_{2}\right.$, where $M=W, M o$ and $\left.X=S e, S\right)$ are indirect bandgap semiconductors composed of a two-dimensional hexagonal lattice. As the material thickness is reduced to a single monolayer, TMDs transition to a direct gap semiconductor with band edges at the $K$ and $K^{\prime}$ momentum valleys at the corners of the Brillouin zone (see Fig. 1). ${ }^{2,3}$ In TMDs the unique combination of broken inversion symmetry, time-reversal symmetry, and strong spin-orbit interaction leads to coupled spin and valley physics. ${ }^{4,5}$ At the $K$

$\dagger$ Send Correspondence To: galan.moody@gmail.com

$\ddagger$ Present Address: National Institute of Standards and Technology, Boulder, CO 80305, USA

Ultrafast Phenomena and Nanophotonics XX, edited by Markus Betz, Abdulhakem Y. Elezzabi, Proc. of SPIE Vol. 9746, 97461T · C 2016 SPIE · CCC code: 0277-786X/16/\$18 doi: $10.1117 / 12.2209203$ 
and $K^{\prime}$ valleys, the magnetic moment and electronic spin orientation are coupled to the valley pseudospin degree of freedom, resulting in chiral optical selection rules.

The large carrier effective masses and reduced dielectric screening in two dimensions strongly enhance Coulomb interactions between quasiparticles in monolayer TMDs compared to conventional semiconductors. Enhanced many-body effects lead to tightly bound exciton states (Coulomb-bound electron-hole pairs) with a Bohr radius of $\sim 1 \mathrm{~nm}$ and a binding energy on the order of $\sim 500 \mathrm{meV}^{6-8}$ In addition to excitons, TMDs host an array of many-body states including tightly bound biexcitons (excitonic molecules) ${ }^{9-11}$ trions (charged excitons), ${ }^{12-14}$ coupled exciton-trion states, ${ }^{15,16}$ localized excitons, ${ }^{17-19}$ and exciton-polaritons. ${ }^{20}$ The optical spectra of monolayer TMDs are dominated by excitons, which inherit the circularly polarized optical selection rules of the bands at the $K$ and $K^{\prime}$ valleys (valley excitons). ${ }^{21-23}$ The robust optical selection rules are illustrated by the low-temperature $(10 \mathrm{~K})$ circularly polarized photoluminescence spectrum shown in Fig. 1(c) for monolayer $\mathrm{WSe}_{2}$ grown on a sapphire substrate through chemical vapor deposition. ${ }^{24}$ The spectrum features two peaks corresponding to the lowest-energy $A$-exciton $(X)$ at $\sim 1720 \mathrm{meV}$ and defect/impurity-bound excitons $(L)$ near $\sim 1620 \mathrm{meV} .{ }^{25}$ After optical excitation of excitons in the $K$ valley using $\sigma+$ polarized light, emission primarily occurs from the same valley $(\sigma+/ \sigma+$ curve compared to $\sigma+/ \sigma-$ curve). The degree of valley polarization, defined as $\rho_{c}=\left(I_{+}-I_{-}\right) /\left(I_{+}+I_{-}\right)$, where $I_{+}\left(I_{-}\right)$corresponds to the detected intensity for $\sigma+$ $(\sigma-)$ polarization, is $\approx 70 \%$ for the exciton transition, indicating robust valley polarization during the exciton lifetime.

Alternatively, excitons in both $K$ and $K^{\prime}$ valleys can be excited simultaneously using linearly polarized excitation. The $\sigma+$ and $\sigma-$ components of the excitation field excite hot carriers in both valleys, which subsequently cool to the exciton states. During this process, the carrier wavefunctions maintain a fixed phase relationship (initialized by the linear excitation field), and this phase is robust against intra- and inter-valley carrier scattering and electron-hole exchange. As a result, the emitted photons at the exciton transition are linearly polarized primarily along the excitation field polarization direction-a signature of exciton valley coherence. ${ }^{25-27}$ Reports of the degree of linear polarization, defined as $\rho_{L}=\left(I_{H}-I_{V}\right) /\left(I_{H}+I_{V}\right)$, where $I_{H}\left(I_{V}\right)$ corresponds to the detected intensity for $H(V)$ polarization, have been as high as $\approx 50 \%$.

One implication of the high degree of valley polarization and valley coherence is the ability to control the valley pseudospin using polarized optical excitation, which provides novel opportunities for opto-electronic, spintronic, and valleytronic applications; ${ }^{28}$ however, in order to leverage the unique optical properties of monolayer

(a)

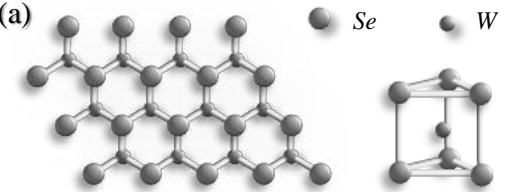

(b)
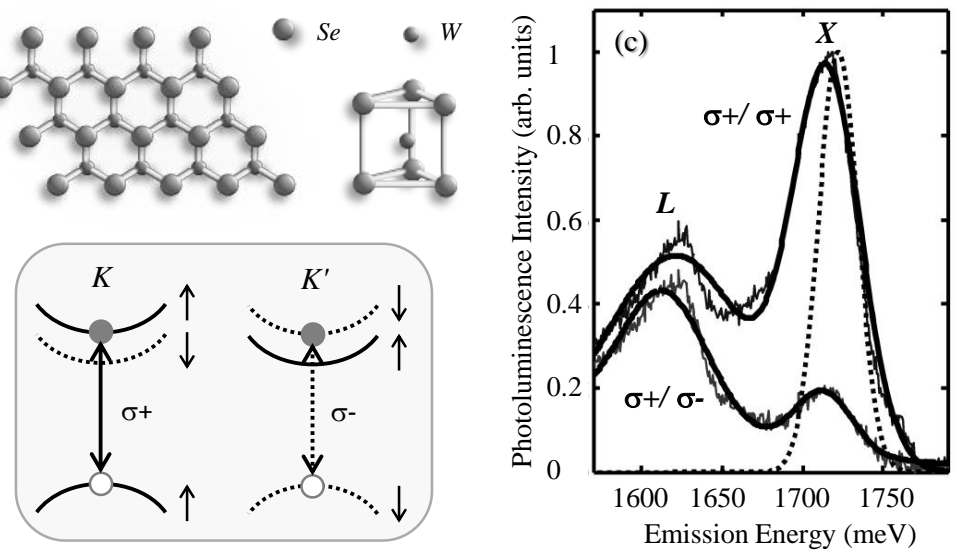

Figure 1. Optical and electronic properties of monolayer $\mathrm{WSe}_{2}$. (a) Schematic of the two-dimensional hexagonal lattice. Direct bandgaps form at the $K$ and $K^{\prime}$ valleys at the corners of the first Brillouin zone. (b) The orbital magnetic moment and electronic spin degrees of freedom are coupled to the valley pseudospin, resulting in circularly polarized optical selection rules for the lowest energy $A$-exciton. Excitons in the $K\left(K^{\prime}\right)$ valley are coupled to $\sigma+(\sigma-)$ polarized light. (c) Circularly polarized photoluminescence spectrum at $10 \mathrm{~K}$ for $\sigma+$ polarized excitation featuring two inhomogeneously broadened peaks associated with the exciton $(X)$ and defect/impurity-bound excitons $(L)$. The exciton degree of circular polarization is $70 \%$. The excitation spectrum for the nonlinear spectroscopy experiments is depicted by the dashed curve. 
TMDs, a clear understanding of the exciton recombination and decoherence mechanisms is required. Insight into the exciton recombination and valley relaxation dynamics has been obtained using time-resolved photoluminescence, ${ }^{29-35}$ Kerr rotation, ${ }^{36}$ and pump-probe spectroscopies. ${ }^{37-40}$ However, these techniques probe the incoherent population, spin, and valley dynamics and thus do not provide information regarding the coherent quantum dynamics of excitons in TMDs. In this work, we examine coherent dynamics of excitons in monolayer $\mathrm{WSe}_{2}$ using optical two-dimensional coherent spectroscopy (2DCS)-a three-pulse four-wave mixing (FWM) technique. This technique has been a particularly powerful tool for studying excitonic transitions in conventional semiconductors ${ }^{41}$ by providing insight into coupling between exciton resonances in quantum wells ${ }^{42-44}$ and quantum dots ${ }^{45,46}$ revealing the homogeneous linewidth of inhomogeneously broadened quantum dot ensembles ${ }^{47}$ quantifying many-body effects between excitons, ${ }^{48-50}$ and separating perturbative orders of the nonlinear optical response of quantum systems. ${ }^{51-53}$ 2DCS is employed in this study to extract the exciton homogeneous linewidth and recombination rate in monolayer $\mathrm{WSe}_{2}$. A comparison of the exciton coherence time to the exciton recombination lifetime reveals the intrinsic coherence decay channels, which are attributed to exciton-exciton and exciton-phonon interactions in addition to exciton recombination. ${ }^{54}$

\section{EXPERIMENT}

\subsection{Quantum Dynamics of Excitons}

The parameters characterizing the coherent quantum dynamics of excitons in TMDs are illustrated by the twolevel energy diagram in Fig. 2(a). An exciton with resonance frequency $\omega_{0}$ can radiatively and non-radiatively recombine with a rate $\Gamma_{K}$ (inversely proportional to the exciton lifetime $T_{1}$ ). A coherent superposition of the crystal ground $(|0\rangle)$ and exciton $(|1\rangle)$ states decays with a rate $\gamma$ (inversely proportional to the coherence time $T_{2}$ ), which defines the homogeneous linewidth. The two are related through $\gamma=\Gamma_{K} / 2+\gamma^{*}$, where $\gamma^{*}$ corresponds to elastic pure dephasing processes such as exciton-phonon and exciton-exciton scattering. In TMDs, local potentials arising from impurities, defects, and the substrate can shift the exciton resonance, resulting in an inhomogeneous distribution of exciton frequencies with width $\Gamma_{i n}$ (Fig. 2(b)). Inhomogeneous broadening has concealed the intrinsic homogeneous linewidth of the exciton in most low-temperature optical spectroscopy experiments.

\subsection{Two-Dimensional Coherent Spectroscopy}

We examine monolayer $\mathrm{WSe}_{2}$ flakes $\sim 20 \mu \mathrm{m}$ in size grown using chemical vapor deposition onto a sapphire substrate. ${ }^{24}$ The sample is held in a cold-finger optical cryostat at variable temperature from $6 \mathrm{~K}$ to $50 \mathrm{~K}$. Monolayers are identified through atomic force microscopy (data not shown). A typical photoluminescence spectrum is shown in Fig. 1(c), which enables identification of the exciton resonance at $10 \mathrm{~K}$. The laser excitation spectrum for the nonlinear spectroscopy experiments is depicted by the dashed curve. 2DCS experiments are performed using a sequence of three phase-stabilized, co-circularly polarized laser pulses propagating in the box geometry and separated by delays $t_{1}$ and $t_{2}$ (see Fig. 2(c)). ${ }^{55}$ The interaction of the pulses with the sample generates a FWM signal $\mathbf{E}_{\mathrm{S}}\left(t_{1}, t_{2}, t_{3}\right)$ that radiates as a "photon echo" in the phase-matched direction $\mathbf{k}_{s}=-\mathbf{k}_{1}$ $+\mathbf{k}_{2}+\mathbf{k}_{3}$, where $\mathbf{k}_{i}$ corresponds to the wavevector of the $i^{\text {th }}$ pulse. The signal field $\mathbf{E}_{\mathrm{S}}$ is detected through heterodyne spectral interferometry with a fourth phase-stabilized reference pulse.

By choosing which of the excitation pulses is scanned, different contributions to the nonlinear optical response can be probed that are sensitive to specific quantum dynamics. In these experiments, the first pulse creates an exciton polarization, or coherence, in the $K$ valley that decays with rate $\gamma$. After a time $t_{1}$, the second pulse converts this coherence to an exciton population in the same valley that decays with rate $\Gamma_{K}$. After a time $t_{2}$, the third pulse converts the population in the $K$ valley to an optical coherence that radiates as the FWM signal. The exciton coherence dynamics are measured by stepping the delay $t_{1}$ while holding the delay $t_{2}$ fixed. Fourier transformation of the signal field with respect to $t_{1}$ yields a $2 \mathrm{D}$ spectrum $\mathbf{E}_{\mathrm{S}}\left(\hbar \omega_{1}, t_{2}, \hbar \omega_{3}\right)$, which correlates the "one-quantum" excitation and emission energies of the system during the delays $t_{1}$ and $t_{3}$ (the $\hbar \omega_{3}$ axis is provided by the spectrometer). Alternatively, the delay $t_{1}$ can be held fixed while the delay $t_{2}$ is varied, which is analogous to pump-probe techniques commonly used. These two types of experiments provide information on the exciton optical coherence $\left(T_{2}=\hbar / \gamma\right)$ and recombination times $\left(T_{1}=\hbar / \Gamma_{K}\right)$, respectively. 
(a)

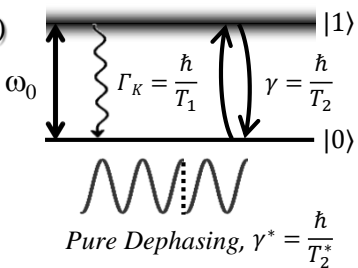

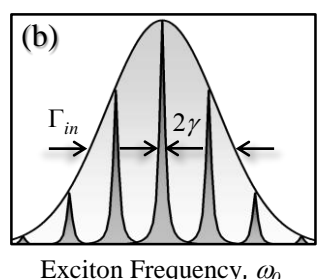

Exciton Frequency, $\omega_{0}$ (c)

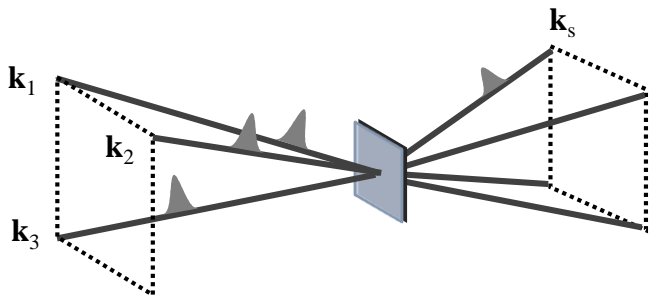

Figure 2. The coherent quantum dynamics of excitons are described using a two-level system shown in (a). Excitons with resonance frequency $\omega_{0}$ can recombine with rate $\Gamma_{K}$ (lifetime $T_{1}$ ). Exciton coherence decays with rate $\gamma$ (coherence time $T_{2}$ ), which is equivalent to the homogeneous linewidth. The two are related through $\gamma=\Gamma_{K} / 2+\gamma^{*}$, where $\gamma^{*}$ is the pure dephasing rate associated with elastic scattering processes. (b) Local potentials due to defects and impurities result in a distribution of exciton frequencies, which appears as inhomogeneous broadening $\left(\Gamma_{i n}\right)$ of the exciton resonance in linear optical spectra. (c) Box geometry used for the three-pulse two-dimensional coherent spectroscopy experiments. The four-wave mixing signal is emitted in the phase-matched direction $\mathbf{k}_{s}=-\mathbf{k}_{1}+\mathbf{k}_{2}+\mathbf{k}_{3}$.

\section{RESULTS AND DISCUSSION}

A typical "one-quantum" 2D spectrum is shown in Fig. 3(a) for a sample temperature of $10 \mathrm{~K}$ and an exciton excitation density of $10^{11} \mathrm{~cm}^{-2}$. The $\hbar \omega_{1}$ axis is plotted as negative excitation energy because the system evolves during the delay $t_{1}$ with opposite phase accumulation relative to that during the delay $t_{3}-$ a consequence of the photon echo time ordering of the excitation pulses. The spectrum features a single peak at the excitation $\left(\hbar \omega_{1}\right)$ and emission $\left(\hbar \omega_{3}\right)$ energies of the exciton resonance. The width of the peak along the diagonal dashed line corresponds to the exciton inhomogeneous broadening in the sample, whereas the half-width at half-maximum (HWHM) of a square root of Lorentzian fit function to a cross-diagonal slice provides a measure of the exciton homogeneous linewidth, ${ }^{56}$ illustrated by the lineshape in Fig. 3(b). At this temperature and excitation density, we find that $\gamma=2.7 \mathrm{meV}\left(T_{2}=250 \mathrm{fs}\right)$.

Additional experiments are performed for exciton excitation densities up to $\approx 10^{12} \mathrm{~cm}^{-2}$. The measured homogeneous linewidth at each density is shown in Fig. 3(c) for a sample temperature of $10 \mathrm{~K}$. The linewidth (points) increases linearly by more than a factor of two-a clear signature of excitation-induced dephasing (EID) arising from elastic exciton-exciton scattering. EID can be described by $\gamma\left(N_{X}\right)=\gamma_{0}+\gamma_{X} N_{X}$, where $N_{X}$ is the exciton population density, $\gamma_{0}$ is the zero-density linewidth, and $\gamma_{X}$ is an exciton elastic interaction parameter. A fit to the data (solid line) yields $\gamma_{X}=2.7 \times 10^{-12} \mathrm{meV} \mathrm{cm}{ }^{2}$ and $\gamma_{0}=2.3 \mathrm{meV}\left(T_{2}=290 \mathrm{fs}\right.$ ). Compared to semiconductor systems of different composition and dimensionality (such as GaAs and ZnSe quantum wells), broadening due to EID measured here $\left(\equiv \gamma-\gamma_{0}\right)$, when normalized to the exciton Bohr radius, is nearly an order of magnitude larger. ${ }^{57,58}$ Enhanced exciton-exciton interactions in TMDs are attributed to reduced dielectric screening in the 2D monolayers.

The role of phonons on exciton dephasing is examined by repeating the excitation density dependent measurements at various temperatures from $6 \mathrm{~K}$ and $50 \mathrm{~K}$. Zero-density linewidths are extracted from linear fits to the linewidth data at each temperature and are shown in Fig. 3(d). The linewidth increases to $\gamma_{0}=4.4 \mathrm{meV}$ $\left(T_{2}=150 \mathrm{fs}\right)$ at $50 \mathrm{~K}$. The linear increase with temperature is indicative of exciton scattering from acoustic phonons with energy much smaller than $k_{B} T$, where $k_{B}$ is the Boltzmann constant and $T$ is the sample temperature. The data are modeled by $\gamma_{0}(T)=\gamma_{0}(0)+\gamma^{\prime} T$, where $\gamma^{\prime}$ is the exciton-phonon coupling strength. A fit to the data yields $\gamma^{\prime}=60 \mu \mathrm{eV} \mathrm{K}^{-1}$, which is nearly an order of magnitude larger compared to conventional semiconductor quantum wells ${ }^{57,58}$ and is a factor of two larger than excitons in bulk TMD InSe. ${ }^{59}$ Extrapolated to zero density and zero temperature, the exciton homogeneous linewidth is $\gamma_{0}(0)=1.6 \mathrm{meV}\left(T_{2}=410 \mathrm{fs}\right)$.

Insight into the remaining exciton decoherence mechanisms after eliminating EID and acoustic phonon interaction effects is obtained by scanning the delay $t_{2}$ while holding the delay $t_{1}$ fixed in order to probe the exciton recombination dynamics as described previously. At a sample temperature of $6 \mathrm{~K}$ and exciton excitation density of $\approx 10^{11} \mathrm{~cm}^{-2}$, we measure a biexponential decay response with fast and slow recombination rates of $\Gamma_{\text {fast }}=3.2$ $\mathrm{meV}$ and $\Gamma_{\text {slow }}=40 \mu \mathrm{eV}$, respectively (data not shown). The amplitude of the fast decay component is approximately a factor of five larger than that of the slow component. We attribute the fast decay to relaxation of the 

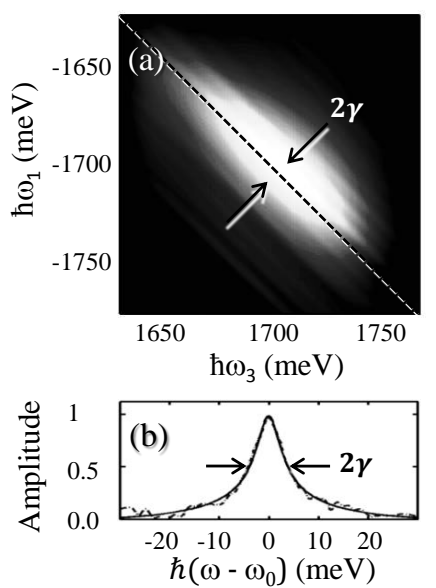
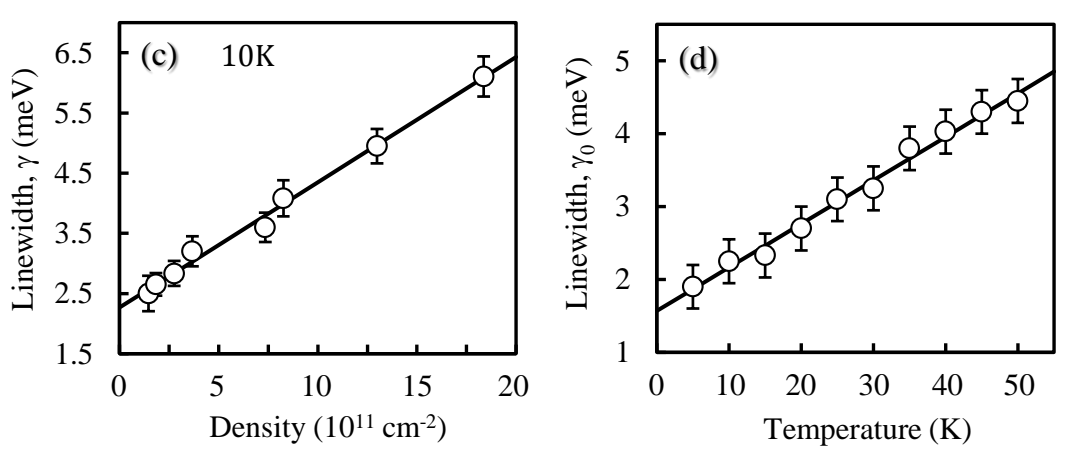

Figure 3. (a) Two-dimensional "one-quantum" rephasing spectrum correlating the excitation $\left(\hbar \omega_{1}\right)$ and emission $\left(\hbar \omega_{3}\right)$ energies of the $A$-exciton. The spectrum features a single peak centered at the exciton absorption energy, which is elongated along the dashed diagonal line due to inhomogeneous broadening. The half-width at half-maximum of a crossdiagonal slice provides a measure of the homogeneous linewidth $\gamma$, shown in (b). (c) The homogeneous linewidth increases linearly with exciton density, shown for $10 \mathrm{~K}$. (d) The zero-density homogeneous linewidth, obtained by repeating the measurements in (c) at various temperatures, increases linearly with temperature, shown up to 50 K. The zero-density, zero-temperature linewidth extrapolated from the fit is $\gamma_{0}=1.6 \mathrm{meV}$.

bright exciton. The biexponential decay implies that additional states beyond a simple two-level system picture contribute to the recombination dynamics. The slow component might arise from the relaxation of energetically degenerate localized or dark excitons with a slower recombination rate or from repopulation of the bright exciton state. ${ }^{35}$ The slow component could also be partially attributed to a thermal distribution of excitons with non-zero center-of-mass momentum. The average response of the thermalized exciton distribution could give rise to an effective lifetime on the order ten picoseconds at cryogenic temperatures. ${ }^{29,60}$ Interestingly, the fact that $\gamma=\Gamma_{K} / 2$ at low temperature and excitation density implies that elastic pure dephasing processes are absent or slow, i.e. $\gamma^{*}=0$. Therefore exciton decoherence is limited strictly by exciton population recombination for this sample and under these experimental conditions.

In summary, two-dimensional coherent spectroscopy has been implemented to examine the exciton quantum dynamics in monolayer $\mathrm{WSe}_{2}$. Recombination and coherence times of excitons excited in a single valley are probed through a combination of different variations of two-dimensional coherent spectroscopy. We show that exciton-exciton and exciton-phonon interactions act as effective optical decoherence channels as the sample temperature increases above $6 \mathrm{~K}$.

\section{ACKNOWLEDGMENTS}

The experimental work at UT-Austin is supported partially by AFOSR grant number FA9550-10-1-0022, NSF DMR-1306878, and Welch Foundation F-1662. X.L. acknowledges support from a Humboldt fellowship. L.J.L. thanks support from KAUST Saudi Arabia, Academia Sinica Taiwan, and AOARD-134137 USA. C.H.C. thanks support from Ministry of Science and Technology Taiwan (MOST 104-2218-E-035-010 and 104-2628-E-035-002MY3). G.C. and X.X. are supported by DoE BES (DE-SC0008145 and DE-SC0012509). The Berlin group is thankful to the German Science Foundation within the collaborative research center 951.

\section{REFERENCES}

[1] Novoselov, K., Jiang, D., Schedin, F., Booth, T., Khotkevich, V., Morozov, S., and Geim, A., "Twodimensional atomic crystals," Proc. Natl. Acad. Sci. 102, 10451-10453 (2005).

[2] Mak, K., Lee, C., Hone, J., Shan, J., and Heinz, T., "Atomically thin mos$_{2}$ : A new direct-gap semiconductor," Phys. Rev. Lett. 105, 136805 (2010). 
[3] Splendiani, A., Sun, L., Zhang, Y., Li, T., Kim, J., Chim, C.-Y., Galli, G., and Wang, F., "Emerging photoluminescence in monolayer $\operatorname{mos}_{2}, "$ Nano Lett. 10, 1271-1275 (2010).

[4] Xiao, D., Liu, G.-B., Feng, W., Xu, X., and Yao, W., "Coupled spin and valley physics in monolayers of $\operatorname{mos}_{2}$ and other group-vi dichalcogenides," Phys. Rev. Lett. 108, 196802 (2012).

[5] Xu, X., Yao, W., Xiao, D., and Heinz, T., "Spins and pseudospins in layered transition metal dichalcogenides," Nat. Phys. 10, 343-350 (2014).

[6] Chernikov, A., Berkelbach, T., Hill, H., Rigosi, A., Li, Y., Aslan, O., Reichman, D., Hybertsen, M., and Heinz, T., "Exciton binding energy and nonhydrogenic rydberg series in monolayer $\mathrm{ws}_{2}, "$ Phys. Rev. Lett. 113, 076802 (2014).

[7] Ugeda, M., Bradley, A., Shi, S.-F., da Jornada, F., Zhang, Y., Qiu, D., Ruan, W., Mo, S.-K., Hussain, Z., Shen, Z.-X., Wang, F., Louie, S., and Crommie, M., "Giant bandgap renormalization and excitonic effects in a monolayer transition metal dichalcogenide semiconductor," Nat. Mater. 13, 1091-1095 (2014).

[8] He, K., Kumar, N., Zhao, L., Wang, Z., Mak, K., Zhao, H., and Shan, J., "Tightly bound excitons in monolayer wse 2 ," Phys. Rev. Lett. 113, 026803 (2014).

[9] You, Y., Zhang, X.-X., Berkelbach, T., Hybertsen, M., Reichman, D., and Heinz, T., "Observation of biexcitons in monolayer wse 2 ," Nat. Phys. 11, 477-481 (2015).

[10] Shang, J., Shen, X., Cong, C., Peimyoo, N., Cao, B., Eginligil, M., and Yu, T., "Observation of excitonic fine structure in a 2d transition-metal dichalcogenide semiconductor," ACS Nano 9, 647-655 (2015).

[11] Sie, E., Frenzel, A., Lee, Y.-H., Kong, J., and Gedik, N., "Intervalley biexcitons and many-body effects in monolayer mos $_{2}, "$ Phys. Rev. B 92, 125417 (2015).

[12] Mak, K., He, K., Lee, C., Lee, G., Hone, J., Heinz, T., and Shan, J., "Tightly bound trions in monolayer $\operatorname{mos}_{2}, "$ Nat. Mater. 12, 207-211 (2013).

[13] Ross, J., S., W., Yu, H., Ghimire, N., Jones, A., Aivazian, G., Yan, J., Mandrus, D., Xiao, D., Yao, W., and $\mathrm{Xu}, \mathrm{X}$., "Electrical control of neutral and charged excitons in a monolayer semiconductor," Nat. Commun. 4, 1474 (2013).

[14] Berkelbach, T., Hybertsen, M., and Reichman, D., "Theory of neutral and charged excitons in monolayer transition metal dichalcogenides," Phys. Rev. B 88, 045318 (2013).

[15] Singh, A., Moody, G., Wu, S., Wu, Y., Ghimire, N., Yan, J., Mandrus, D., Xu, X., and Li, X., "Coherent electronic coupling in atomically thin mose $_{2}, "$ Phys. Rev. Lett. 112, 216804 (2014).

[16] Singh, A., Moody, G., Tran, K., Scott, M., Overbeck, V., Berghauser, G., Schaibley, J., Seifert, E., Pleskot, D., Gabor, N., Yan, J., Mandrus, D., Richter, M., Malic, E., Xu, X., and Li, X., "Trion formation dynamics in monolayer transition metal dichalcogenides," Phys. Rev. B 93, 041401 (2016).

[17] Srivastava, A., Sidler, M., Allain, A., Lembke, D., Kis, A., and Imamoglu, A., "Optically active quantum dots in monolayer wse $2, "$ Nat. Nanotech. 10, 491-496 (2015).

[18] Chakraborty, C., Kinnischtzke, L., Goodfellow, K., Beams, R., and Vamivakas, A., "Voltage-controlled quantum light from an atomically thin semiconductor," Nat. Nanotech. 10, 507-511 (2015).

[19] Koperski, M., Nogajewski, K., Arora, A., Cherkez, V., Mallet, P., Veuillen, J.-Y., Marcus, J., Kossacki, P., and Potemski, M., "Single photon emitters in exfoliated wse 2 structures," Nat. Nanotech. 10, 503-506 (2015).

[20] Liu, X., Galfsky, T., Sun, Z., Xia, F., Lin, E.-C., Lee, Y.-H., Kena-Cohen, S., and Menon, V., "Strong light-matter coupling in two-dimensional atomic crystals," Nat. Photon. 9, 30-34 (2015).

[21] Mak, K., He, K., Shan, J., and Heinz, T., "Control of valley polarization in monolayer $\operatorname{mos}_{2}$ by optical helicity," Nat. Nanotech. 7, 494-498 (2012).

[22] Zeng, H., Dai, J., Yao, W., Xiao, D., and Cui, X., "Valley polarization in $\operatorname{mos}_{2}$ monolayers by optical pumping," Nat. Nanotech. 7, 490-493 (2012).

[23] Cao, T., Wang, G., Han, W., Ye, H., Zhu, C., Shi, J., Niu, Q., Tan, P., Wang, E., Liu, B., and Feng, J., "Valley-selective circular dichroism of monolayer molybdenum disulphide," Nat. Commun. 3, 887 (2012).

[24] Huang, J.-K., Pu, J., Hsu, C.-L., Chiu, M.-H., Juang, Z.-Y., Chang, Y.-H., Chang, W.-H., Iwasa, Y., Takenobu, T., and Li, L.-J., "Large-area synthesis of highly crystalline wse $_{2}$ monolayers and device applications," ACS Nano 8, 923-930 (2014). 
[25] Wang, G., Marie, X., Gerber, I., Amand, T., Lagarde, D., Bouet, L., Vidal, M., Balocchi, A., and Urbaszek, B., "Giant enhancement of the optical second-harmonic emission of $\mathrm{wse}_{2}$ monolayers by laser excitation at exciton resonances," Phys. Rev. Lett. 114, 097403 (2015).

[26] Jones, A., Yu, H., Ghimire, N., Wu, S., Aivazian, G., Ross, J., Zhao, B., Yan, J., Mandrus, D., Xiao, D., Yao, $\mathrm{W}$., and $\mathrm{Xu}, \mathrm{X}$., "Optical generation of excitonic valley coherence in monolayer wse $_{2}$," Nat. Nanotech. 8, 634-638 (2013).

[27] Hao, K., Moody, G., Wu, F., Dass, C., Xu, L., Chen, C.-H., Li, M.-Y., Li, L.-J., MacDonald, A., and Li, X., "Direct measurements of exciton valley coherence in monolayer $\mathrm{wse}_{2}$," arXiv: 1509.08810 .

[28] Jariwala, D., Sangwan, V., Lauhon, L., Marks, T., and Hersam, M., "Emerging device applications for semiconducting two-dimensional transition metal dichalcogenides," ACS Nano 8, 1102-1120 (2014).

[29] Korn, T., Heydrich, S., Hirmer, M., Schmutzler, J., and Schuller, C., "Low-temperature photocarrier dynamics in monolayer $\operatorname{mos}_{2}, "$ Appl. Phys. Lett. 99, 102109 (2011).

[30] Mouri, S., Miyauchi, Y., Toh, M., Zhao, W., Eda, G., and Matsuda, K., "Nonlinear photoluminescence in atomically thin layered $\mathrm{wse}_{2}$ arising from diffusion-assisted exciton-exciton annihilation," Phys. Rev. B 90, 155449 (2014).

[31] Wang, G., Bouet, L., Lagarde, D., Vidal, M., Balocchi, A., Amand, T., Marie, X., and Urbaszek, B., "Valley dynamics probed through charged and neutral exciton emission in monolayer wse 2 ," Phys. Rev. $B$ 90, 075413 (2014).

[32] Lagarde, D., Bouet, L., Marie, X., Zhu, C., Liu, B., Amand, T., Tan, P., and Urbaszek, B., "Carrier and polarization dynamics in monolayer $\operatorname{mos}_{2}, "$ Phys. Rev. Lett. 112, 047401 (2014).

[33] Wang, G., Palleau, E., Amand, T., Tongay, S., Marie, X., and Urbaszek, B., "Polarization and time-resolved photoluminescence spectroscopy of excitons in mose 2 monolayers," Appl. Phys. Lett. 106, 112101 (2015).

[34] Amani, M., Lien, D.-H., Kiriya, D., Xiao, J., Azcatl, A., Noh, J., Madhvapathy, S., Addou, R., KC, S., Dubey, M., Cho, K., Wallace, R., Lee, S.-C., He, J.-H., Ager III, J., Zhang, X., Yablonovitch, E., and Javey, A., "Near-unity photoluminescence quantum yield from $\operatorname{mos}_{2}$," Science 350, 1065-1068 (2015).

[35] Zhang, X.-X., You, Y., Frank Zhao, S., and Heinz, T., "Experimental evidence for dark excitons in monolayer wse $_{2}, "$ Phys. Rev. Lett. 115, 257403 (2015).

[36] Glazov, M., Ivchenko, E., Wang, G., Amand, T., Marie, X., Urbaszek, B., and Liu, B., "Spin and valley dynamics of excitons in transition metal dichalcogenide monolayers," Phys. Status Solidi B 252, 2349-2362 (2015).

[37] Shi, H., Yan, R., Bertolazzi, S., Brivio, J., Gao, B., Kis, A., Jena, D., Xing, H., and Huang, L., "Exciton dynamics in suspended monolayer and few-layer mos $_{2} 2$ d crystals," ACS Nano 7, 1072-1080 (2013).

[38] Wang, H., Zhang, C., and Rana, F., "Surface recombination limited lifetimes of photoexcited carriers in few-layer transition metal dichalcogenide $\operatorname{mos}_{2}, "$ Nano Lett. 15, 8204-8210 (2015).

[39] Cui, Q., Ceballos, F., Kumar, N., and Zhao, H., "Transient absorption microscopy of monolayer and bulk wse $_{2}, "$ ACS Nano 8, 2970-2976 (2014).

[40] Sun, D., Rao, Y., Reider, G., Chen, G., You, Y., Brezin, L., Harutyunyan, A., and Heinz, T., "Observation of rapid exciton-exciton annihilation in monolayer molybdenum disulfide," Nano Lett. 14, 5625-5629 (2014).

[41] Cundiff, S., Bristow, A., Siemens, M., Li, H., Moody, G., Karaiskaj, D., Dai, X., and Zhang, T., "Optical 2-d fourier transform spectroscopy of excitons in semiconductor nanostructures," IEEE J. Sel. Top. Quantum Electron. 18, 318-328 (2012).

[42] Davis, J., Hall, C., Dao, L., Nugent, K., Quiney, H., Tan, H., and Jagadish, C., "Three-dimensional electronic spectroscopy of excitons in asymmetric double quantum wells," J. Chem. Phys. 135, 044510 (2011).

[43] Nardin, G., Moody, G., Singh, R., Autry, T., Li, H., Morier-Genoud, F., and Cundiff, S., "Coherent excitonic coupling in an asymmetric double ingaas quantum well arises from many-body effects," Phys. Rev. Lett. 112, 046402 (2014).

[44] Moody, G., Akimov, I., Li, H., Singh, R., Yakovlev, D., Karczewski, G., Wiater, M., Wojtowicz, T., Bayer, M., and Cundiff, S., "Coherent coupling of excitons and trions in a photoexcited cdte/cdmgte quantum well," Phys. Rev. Lett. 112, 097401 (2014).

[45] Kasprzak, J., Patton, B., Savona, V., and Langbein, W., "Coherent coupling between distant excitons revealed by two-dimensional nonlinear hyperspectral imaging," Nat. Photon. 5, 57-63 (2011). 
[46] Moody, G., Siemens, M., Bristow, A., Dai, X., Bracker, A., Gammon, D., and Cundiff, S., "Exciton relaxation and coupling dynamics in a gaas/algaas quantum well and quantum dot ensemble," Phys. Rev. $B$ 83, 245316 (2011).

[47] Moody, G., Siemens, M., Bristow, A., Dai, X., Karaiskaj, D., Bracker, A., Gammon, D., and Cundiff, S., "Exciton-exciton and exciton-phonon interactions in an interfacial gaas quantum dot ensemble," Phys. Rev. $B$ 83, 115324 (2011).

[48] Li, X., Zhang, T., Borca, C., and Cundiff, S., "Many-body interactions in semiconductors probed by optical two-dimensional fourier transform spectroscopy," Phys. Rev. Lett. 96, 57406 (2006).

[49] Moody, G., Singh, R., Li, H., Akimov, I., Bayer, M., Reuter, D., Wieck, A., Bracker, A., Gammon, D., and Cundiff, S., "Influence of confinement on biexciton binding in semiconductor quantum dot eensemble measured with two-dimensional spectroscopy," Phys. Rev. B 87, 041304 (2013).

[50] Dey, P., Paul, J., Moody, G., Stevens, C., Glikin, N., Kovalyuk, Z., Kudrynskyi, Z., Romero, A., Cantarero, A., Hilton, D., and Karaiskaj, D., "Biexciton formation and exciton coherent coupling in layered gase," $J$. Chem. Phys. 142, 212422 (2015).

[51] Turner, D. and Nelson, K., "Coherent measurements of high-order electronic ccorrelation in quantum wells," Nature 466, 1089-1092 (2010).

[52] Moody, G., Singh, R., Li, H., Akimov, I., Bayer, M., Reuter, D., Wieck, A., and Cundiff, S., "Fifthorder nonlinear optical response of excitonic states in an inas quantum dot ensemble measured with twodimensional spectroscopy," Phys. Rev. B 87, 045313 (2013).

[53] Fras, F., Mermillod, Q., Nogues, G., Hoarau, C., Schneider, C., Kamp, M., Hofling, S., Langbein, W., and Kasprzak, J., "Multi-wave coherent control of a solid state single emitter," arXiv:1509.07690 .

[54] Moody, G., Dass, C., Hao, K., Chen, C.-H., Li, L.-J., Singh, A., Tran, K., Clark, G., Xu, X., Berghauser, G., Malic, E., Knorr, A., and Li, X., "Intrinsic homogeneous linewidth and broadening mechanisms of excitons in monolayer transition metal dichalcogenides," Nat. Commun. 6, 8315 (2015).

[55] Bristow, A., Karaiskaj, D., Dai, X., Zhang, T., Carlsson, C., Hagen, K., Jimenez, R., and Cundiff, S., "A versatile ultrastable platform for optical multidimensional fourier-transform spectroscopy," Rev. Sci. Instrum. 80, 073108 (2009).

[56] Siemens, M., Moody, G., Li, H., Bristow, A., and Cundiff, S., "Resonance lineshapes in two-dimensional fourier transform spectroscopy," Opt. Express 18, 17669-17708 (2010).

[57] Honold, A., Schultheis, L., Kuhl, J., and Tu, C., "Collision broadening of two-dimensional excitons in a gaas single quantum well," Phys. Rev. B 40,6442-6445 (1989).

[58] Wagner, H., Schatz, A., Maier, R., Langbein, W., and Hvam, J., "Coherent optical nonlinonlinear and phase relaxation of quasi-three-dimensional and quasi-two-dimensional excitons in znsse/znse structures," Phys. Rev. B 56, 12581-12588 (1997).

[59] Dey, P., Paul, J., Glikin, N., Kovalyuk, Z., Kudrynskyi, Z., Romero, A., and Karaiskaj, D., "Mechanism of excitonic dephasing in layered inse crystals," Phys. Rev. B 89, 125128 (2014).

[60] Palummo, M., Bernardi, M., and Grossman, J., "Exciton radiative lifetimes in two-dimensional transition metal dichalcogenides," Nano Lett. 15, 2794-2800 (2015). 